\title{
Primary orbital liposarcoma: clinical and computed tomographic features
}

\author{
Alan A McNab, Ivan Moseley
}

\begin{abstract}
Liposarcoma of the orbit is rare. The clinical and radiological characteristics of two cases, of myxoid and pleomorphic types, are reported. In neither case was the histological diagnosis evident before surgery.
\end{abstract}

Primary orbital liposarcoma is extremely rare. Sixteen cases, mostly myxoid and none definitely identified as pleomorphic, have been reported in detail in the literature. The two patients described here are the fourth and fifth to be seen in the Orbital Clinic at Moorfields Eye Hospital since 1982. The preceding patients, all of whom had myxoid tumours, have been reported on previously. ${ }^{1}$ To our knowledge the clinical and radiological features of pleomorphic liposarcoma arising in the orbit are described for the first time.

\section{Case reports}

CASE 1

A 69-year-old woman complained of progressive right proptosis over six months. She had vertical diplopia and difficulty in seeing colours clearly with the right eye. Her general health was excellent.

Her best corrected vision was $6 / 9$ in the right eye and $6 / 6$ left. She made four errors on the Ishihara test plates with the right eye, and was thought to have an afferent pupillary defect. The globe was displaced $9 \mathrm{~mm}$ forwards and $4 \mathrm{~mm}$ downwards. Ocular motility was restricted,

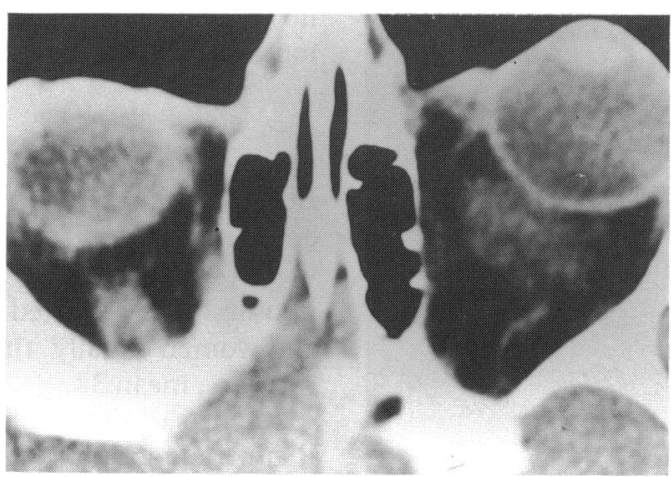

Figure $1 A$

Moorfields Eye Hospital, City Road, London

EC1V 2PD

A A $\mathrm{McNab}$

I Moseley

Correspondence to

Dr I Moseley.

Accepted for publication

25 January 1990
Figure 1: Case 1. CT with intravenous contrast medium. $A$ xial $(A)$ and direct coronal $(B)$ sections show a well defined, rounded, intraconal mass lying above the globe, which is not distorted. The mass extends posteriorly above the optic nerve $(C)$. The orbit is not enlarged. The radiographic density of the mass is uniform, being intermediate between that of the
(C). The orbit is not enlarged. The radiographic density vitreous and that of normal orbital fat - that is, it appears less dense than water. The more anterior coronal image $(B)$ suggests a capsule of higher density.

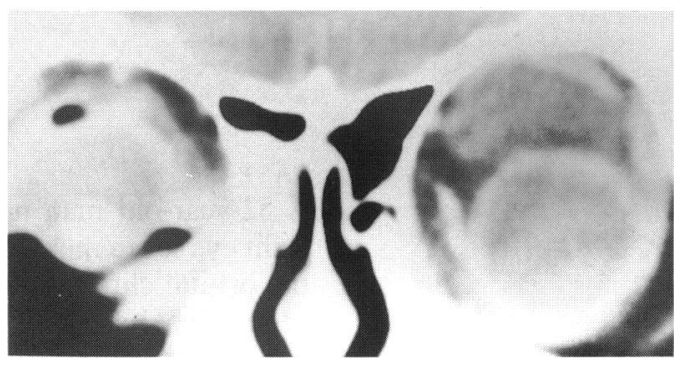

Figure $1 B$

particularly in elevation. A non-tender mass was palpable in the upper orbit. General examination was normal, as was a chest radiograph.

Computed tomography (CT) of the orbits was obtained in sagittal and coronal planes after intravenous injection of iodinated contrast medium (Fig 1). A well defined, rounded intraconal mass of low density was shown, lying just above the globe.

The mass was explored via an upper lid skin incision, the levator palpebrae superioris and superior rectus muscles being retracted medially. Anteriorly the tumour was well circumscribed, but posteriorly it appeared to blend with the orbital fat. It was pale, yellowgrey, and petechial haemorrhages appeared on its surface as it was touched. The incisional biopsy specimen floated in $10 \%$ formaldehyde solution.

Histological examination revealed a tumour with numerous rounded spaces which on frozen section contained lipid. Some cells were spindle shaped and lay in a mucinous matrix, while others showed a signet ring form. There was nuclear hyperchromatism, scattered cells having enlarged, pleomorphic nuclei; some of the nuclei were vesicular. Numerous capillaries were present throughout the mass. The appearances were typical of a myxoid liposarcoma (Fig 2).

Over the next four weeks the proptosis increased by $2 \mathrm{~mm}$. Orbital exenteration was performed, with removal of the upper lid and adequate clearance beyond the posterior margin of the tumour. There has been no evidence of recurrence for more than a year since surgery.

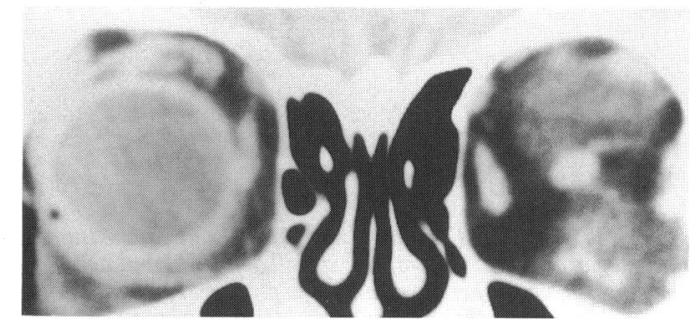

Figure $1 C$ 
Figure 2: Case 1. Myxoid liposarcoma with nuclear hyperchromatism and occasional signet ring cells. The numerous clear spaces contained lipid on frozen section. (Haematoxylin and eosin, $\times 225$.)

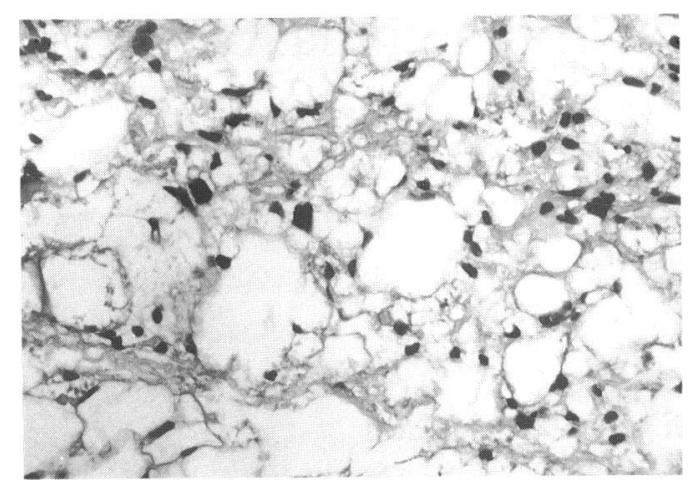

CASE 2

A 52-year-old man noted blurred vision in the right eye seven months before his presentation to the orbital clinic, followed two months later by proptosis. His vision corrected to 6/9, but he had mild disc swelling and $3 \mathrm{~mm}$ of proptosis. This increased to $5 \mathrm{~mm}$ over 3 months, and he developed diplopia on left lateral gaze. He had no pain.

In the orbital clinic his best correctd acuity was $6 / 24$ in the right eye and 6/5 left. He read all the Ishihara plates, but was slower on the right. There was a mild right relative afferent pupil defect. The globe was displaced $5 \mathrm{~mm}$ forwards

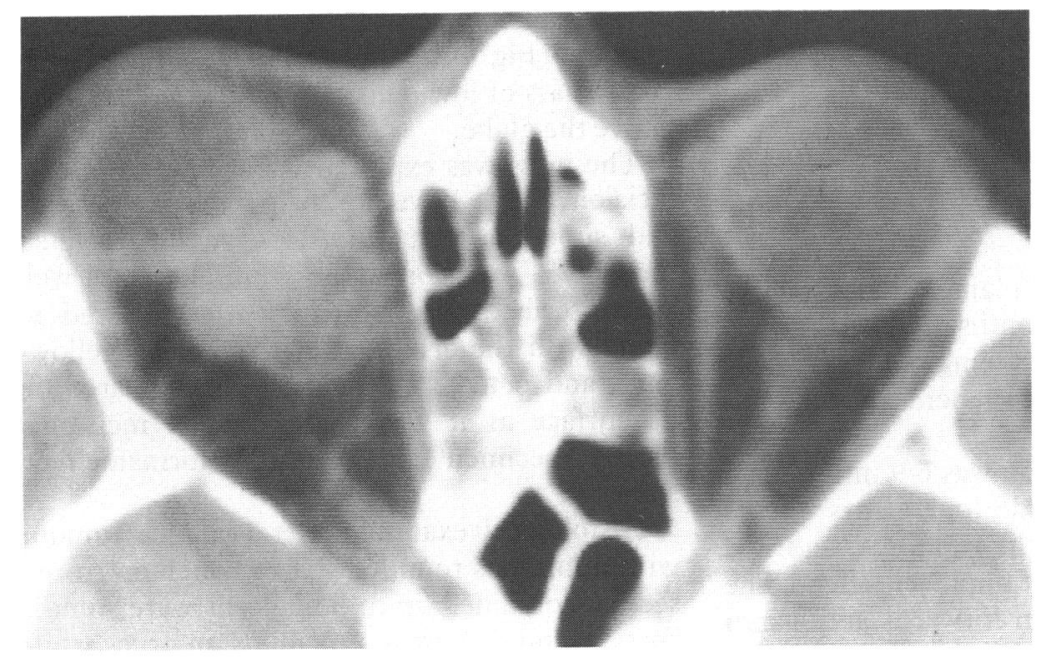

Figure $3 A$

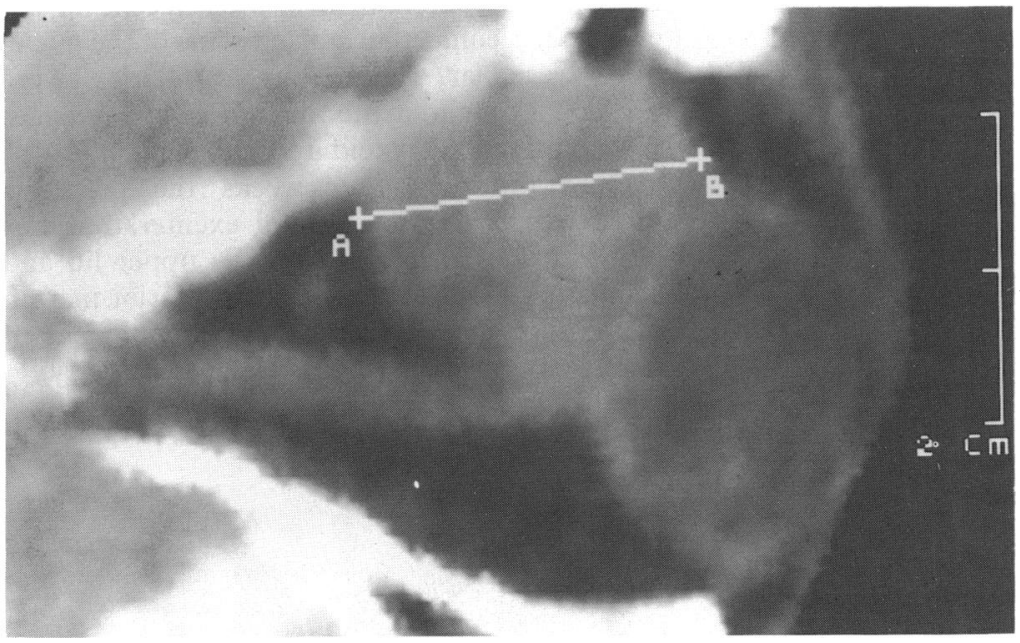

Figure $3 B$

Figure 3: Case 2. CT without intravenous contrast medium. Direct axial $(A)$ and reformatted oblique parasagittal $(B)$ images: a well defined, lobulated mass over $2 \mathrm{~cm}$ in diameter lies within the muscle cone, superomedial to the optic nerve, slightly flattening the globe. The mass is generally isodense with brain; its less dense posterior portion is still denser than the vitreous. The roof of the orbit is very thin, possibly eroded.

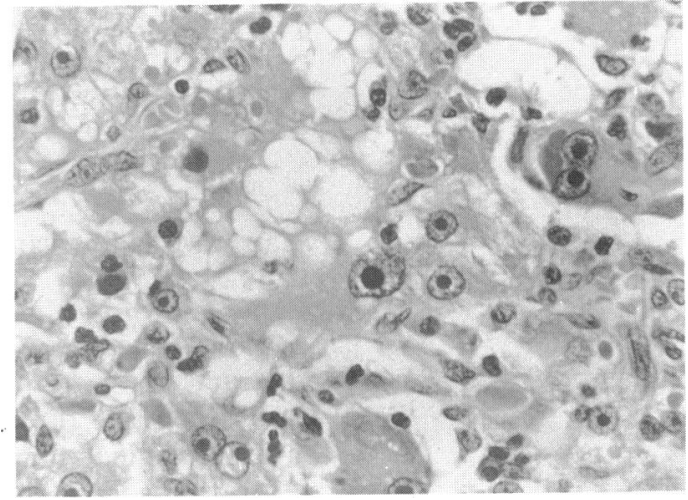

Figure 4: Case 2. Pleomorphic liposarcoma. The nuclei are variable in size, many having large, prominent nucleoli. The clear spaces also contained lipid on frozen section.

(Haematoxylin and eosin preparation, $\times 225$.)

and inferolaterally, with limited elevation and adduction. The optic disc was grossly swollen, with engorged retinal veins, but there were no choroidal folds. The upper lid was full. General examination and chest radiograph were normal.

Plain films of the orbits also showed nothing abnormal, but CT showed a well defined mass within the muscle cone, containing areas of lower density within it (Fig 3).

At lateral orbitotomy a large, purplish, lobulated tumour was found intraconally, abutting the globe, the medial rectus muscle, and the upper surface of the optic nerve. It appeared to be well encapsulated, and was dissected free of the surrounding structures. A large artery entering its posterior pole was divided and the mass was removed in one piece.

Histological examination (Fig 4) disclosed a liposarcoma of extremely variable morphology, with numerous thin walled vessels, lipoblasts, and large, deeply eosinophilic giant cells. Mitotic figures were infrequent, but foci of inflammation and haemorrhage were present. There was no true capsule. The histological diagnosis was therefore pleomorphic liposarcoma.

The orbit was exenterated, together with the scar, but the lids were spared. Examination of the excised tissue showed a single nodule of tumour, less than $1 \mathrm{~mm}$ in diameter, well clear of the resection margin. One year later there was no evidence of local recurrence or distant metastasis.

\section{Discussion}

Because of its rarity liposarcoma does not usually enter into the differential diagnosis in patients with orbital masses. The 16 primary orbital liposarcomas reported in detail ${ }^{1-12}$ affected men and women equally, their ages ranging from 5 to 77 years, mean 34 .

Reports of the results of imaging are sketchy. Plain radiography commonly shows nothing abnormal, but it may show enlargement of the orbit, sometimes considerable, with thinning and inferior displacement of the floor. ${ }^{1}$ Echography may indicate a relatively well defined, multiseptate lesion with non-specific areas of high echogenicity. ${ }^{13}$ In the few patients who have undergone CT, none of whom had pleomorphic tumours, findings have varied from well defined, homogeneous lesions of soft tissue density, not 
evidently fatty, ${ }^{11}{ }^{13}$ to heterogeneous masses containing fat. ${ }^{\prime}$ In two patients CT failed to reveal an identifiable mass, and in one of these $T_{2^{-}}$ weighted magnetic resonance imaging (MRI) showed abnormally high signal from part of the orbital fat, indicating its pathological nature. ${ }^{1}$

In our first patient the clue to the correct diagnosis was the low radiographic density of the lesion: fat is the only tissue component less dense than the water of the vitreous. Choristomas may contain fat, but are not characteristically retrobulbar, and benign lipomas of the orbit are exceedingly rare. Case 2 showed areas of lower density than brain but no clear evidence of fat. There were no radiological features indicating the malignant nature of the tumour in either patient. The short history and documented progression of symptoms and signs are, in retrospect, more suggestive.

Enziger and Weiss ${ }^{14}$ divided primary liposarcomas into myxoid tumours (which form about half) and round cell, well differentiated, and dedifferentiated forms; a pleomorphic type is also described. Myxoid tumours usually have a gelatinous consistency, while poorly differentiated tumours are soft and haemorrhagic, with areas of necrosis and cyst formation. Of the primary orbital lesions reported most were myxoid; one showed predominantly round cells, ${ }^{9}$ while another, described as lipocytic, ${ }^{6}$ might have been categorised as pleomorphic.

Metastasis of liposarcoma to the orbit is also reported, ${ }^{13}$ is the commonest primary sites being the deep connective tissue planes of the thigh or retroperitoneum. In an obese patient like our case 1 it is possible, though unlikely, that a primary tumour went undetected.

The peak incidence of lipomas outside the orbit is between 40 and 60 years of age - that is, later than that of orbital lesions, probably reflecting their less critical location. Patients with myxoid tumours tend to be younger, and males are more commonly affected than females. ${ }^{15}$

Of the 16 well documented cases of primary orbital liposarcoma ${ }^{1-12}$ six resulted in death. The consensus on treatment elsewhere in the body is wide local excision. ${ }^{16-19}$ Radiotherapy affords little benefit in the better differentiated liposarcomas, but successes have been reported with poorly differentiated tumours. ${ }^{16}$ Chemotherapy has not generally been helpful, though Gottlieb et $a l^{20}$ reported remission in three patients.

Both the tumours described here were well defined on CT and appeared relatively well encapsulated at surgery. However, the 'capsule' represents compressed orbital tissues; there is no true capsule between the neoplastic tissue and the normal structures. Three patients previously operated upon in this hospital ${ }^{1}$ have now been followed up for from two to five years without evidence of tumour recurrence or dissemination.

Prognosis of liposarcomas elsewhere in the body has been related to histology. Thus in a series of 77 patients treated by surgical excision, 27 of whom also received radiotherapy, the 10year survival was 12 of 20 with myxoid tumours, but only 1 of 24 with round cell or pleomorphic lesions. Site, size, and adequacy of excision were all important factors. ${ }^{21}$ The rational approach to orbital liposarcoma would therefore appear to be wide local excision, which will usually necessitate exenteration or even midfacial resection. In an elderly patient with a mxyoid or well differentiated tumour local excision is justifiable.

AA McNab was supported by the OPSM fellowship of the Royal Australian College of Ophthalmologists.

1 Lane CM, Wright JE, Garner A. Primary myxoid liposarcoma of the orbit. Br $\mathcal{F}$ Ophthalmol 1988; 72: 912-7.

2 Strauss M. Liposarkom der recten Orbita. Dtsch Med Wochenschr 1911; 37: 239.

3 Stout AP. Liposarcoma - the malignant tumour of lipoblasts. Ann Surg 1944; 119: 86-107.

4 Quéré MA, Camain R, Baylet R. Liposarcome orbitaire. Ann Oculist (Paris) 1963; 196: 994-1003.

5 Cilotti P. Su di particolare forma di liposarcoma dell'orbita con conspicue variazioni morfologiche durante la sua evoluzione. Ann Oftal Oculistica 1964; 90: 325-37.

6 Mortada A. Rare primary orbital sarcomas. Am $\mathcal{f}$ Ophthalmol 1969; 68: 919-25.

7 Henderson JW. Orbital tumors. Philadelphia: Saunders, 1973: $262-7$.

8 Schroeder W, Dastendieck H, von Domarus D. Primäre myxoides Liposarkom der Orbita: klinscher und histopathomyxoides Liposarkom der Orbita: klinscher und histopatho-

9 Kimura Y, Nagato M, Yamabe H. A case of orbital lipoimura Y, Nagato M, Yamabe H. A case of
sarcoma. Fol Ophthalmol fpn 1978; 29: 983-8

10 Jakobiec FA, Jones IS. In: Duane TD, ed. Clinical ophthal mology. Hagerston: Harper and Row, 1982; 2 : 17-9.

11 Naeser P, Mostrom U. Liposarcoma of the orbit: a clinicopathological case report. Br f Ophthalmol 1982; 66: 190-3.

12 Malan P, Baldet P, Arnaud B. Primary orbital liposarcoma Clinicopathological case report and review of the literature. Orbit 1983; 2: 99-105.

13 Nasr AM, Ossoinig KC, Kersten RF, Blodi FC. Standardized echographic-histopathologic correlations in liposarcoma. Am fOphthalmol 1985; 99: 193-200.

14 Enziger FM, Weiss SW. Soft tissue tumors. St Louis: Mosby 1973.

15 Abdalla MI, Ghaly AF, Hosni F. Liposarcoma with orbital metastases. Br f Ophthalmol 1966; 50: 426-8.

16 Enterline HT, Culberson JD, Rochlin DB, Brady LW. Liposarcoma: a clinical and pathological study of 53 cases. Cancer 1960; 13: 932-50.

17 Pack GT, Pierson JC. Liposarcoma: a study of 105 cases Surgery 1954; 36: 687-712.

18 Spittle ME, Newton KA, McKenzie DH. Liposarcoma. A review of 60 cases. Brf Cancer 1970; 24: 696-704.

19 Kinne DW, Chu FCH, Huvos AG, Yagoda A, Fortner JG Treatment of primary and recurrent retroperitoneal liposarcoma. Twenty-five-year experience at Memorial Hospital. Cancer 1973; 31: 53-64.

20 Gottlieb JA, Baker LH, Quagliana JM, et al. Chemotherapy of sarcomas with a combination of adriamycin and dimethyl triazeno imidazole carboxamide. Cancer 1972; 30: 1632-8.

21 Kindblom LG, Angervall L, Svendsen P. Liposarcoma: a clinicopathological, radiographic and prognostic study. Acto Pathol Microbiol Immunol Scand $(A)$ 1975: suppl 253. 